\title{
A Physiologically-Based Pharmacokinetic Model for Vancomycin
}

\author{
by \\ Rebekah White \\ East Tennessee State University \\ whiter1@goldmail.etsu.edu
}

\author{
Advisor \\ Michele Joyner \\ East Tennessee State University \\ joynerm@mail.etsu.edu
}




\begin{abstract}
Vancomycin is an antibiotic used for the treatment of systemic infections. It is given intravenously usually every twelve or twenty-four hours. This particular drug has a medium level of boundedness, with approximately fifty to sixty percent of the drug being free and thus physiologically effective. A physiologically-based pharmacokinetic (PBPK) model was used to better understand the absorption, distribution, and elimination of the drug. Using optimal parameters, the model could be used in the future to test how various factors, such as body mass index (BMI) or excretion levels, might affect the concentration of the antibiotic.
\end{abstract}




\section{Introduction}

The inspiration for this research into the physiologically-based pharmacokinetic (PBPK) modeling of vancomycin was based on work by Forbes [1]. Her research involved the development of a pharmacokinetic model to study ertapenem and the effects BMI has on this drug's physiological properties. The goal of this paper was to explore the stages of developing a PBPK model for vancomycin that would later be used to determine if BMI affected the concentration of vancomycin in the same manner [2]. This paper uses a similar methodology to develop a model for the concentration of vancomycin in the body. This model was developed in stages, where in each stage improvements and alterations were made in order to produce the final model. Specifically, this development included the estimation of several drug specific parameters, the introduction of an infusion coefficient, and ultimately the assumption of nonlinear drug concentrations in the blood. The result of this research was the development of an accurate model that describes the absorption, distribution, and elimination of vancomyin in the body, which could be implemented to study how various factors, such as BMI or gender, affect the physiological properties of vancomycin. This knowledge is useful in determining how a drug should be administered in the future. Specifically, the dosing for an individual of a certain body mass index necessary to avoid renal toxicity or antibiotic resistance is of interest. In Section 2 some specifics about the antibiotic are discussed, as well as the derivation for all the model equations and how the resulting base model's output compares to literature data. Sections 3,4 , and 5 discuss the changes made in order to develop an accurate model. The results of each successive change are given in the respective sections. Section 6 discusses the overall results of this research as well as implications for further research.

\section{THE ANTIBIOTIC AND BASE MODEL}

In order to develop the PBPK model for vacomycin, some drug specific information is required. Vancomycin is a broad spectrum antibiotic and the first line of defense for the treatment of methicillin-resistant Staphylococcuss aureus (MRSA). It is commonly used to treat infections of the bloodstream and skin as well as meningitis [3]. Vancomycin was isolated from soil samples collected in the jungles of Borneo by a missionary, Edmund Kornfeld, in 1953. It was soon realized that bacteria did not build up resistance to the drug as quickly as to other antibiotics such as penicillin. For this reason its name was derived from the word "vanquish" [4]. It is a moderately bound antibiotic, meaning some portion of the drug binds to plasma proteins in the blood and is therefore not pharmacologically effective. The protein binding of vancomycin is approximately $50-60 \%[5,6]$. It is primarily eliminated via the renal route, with

$80 \%-90 \%$ recovered unchanged in urine within 24 hours after administration of a single dose. The remaining drug is eliminated in the stool. There is no apparent metabolism of the drug [5]. The average dose for an adult with healthy 
renal function is 1 gram every 12 or 24 hours. The infusion time is 1 hour.

The first step in creating a PBPK model is to determine the compartments that will be included in the model. In order to do this, the physiological effects the drug has on different organs or tissues of the body must be known. The route of administration as well as how the drug is excreted are also required. This, combined with what aspect of the drug is to be studied, determines which compartments should be included in the model. The route of administration for vancomycin is intravenous. Thus, in our model, blood $(B)$ must be one compartment as this is what will supply the organs of the body with the drug. The effect of BMI on absorption is the ultimate goal of this modeling effort, which means the amount of fat is relevant. Therefore, adipose tissue $(F)$ must also be a compartment. The drug is primarily excreted in the urine, with a small amount being eliminated in the feces. This means the kidney $(K)$ and gut $(G)$ must comprise two compartments in the model. All other tissues $(O T)$ are lumped into one compartment. Information about the other tissues is obtained by subtracting what is calculated regarding those four compartments from what is known about the total body. This grouping of both rapidly and slowly perfused tissues is acceptable for several reasons. First, fat is one of the main slowly perfused tissues and already comprises one compartment of the model. Second, the slowly perfused tissues that would be of concern, the lungs and the liver, are not pharmacokinetically significant. Vancomycin is not readily metabolized by the liver, which indicates the drug does not linger in this tissue. In addition, there is evidence that vancomycin is not able to be transported into the lungs [7]. For these reasons, the grouping of tissues previously laid out is acceptable for this model. Notice this gives us five compartments, blood, fat, kidneys, gut, and other tissues. There are also two types of excretion, that which is in the urine and that which is in the feces.

The next step in creating the PBPK model is to examine how the drug is broken down or activated in the body. This is necessary in order to determine the drug's physiological effects on the body. A portion of the drug is bound to plasma proteins in the body and rendered ineffective. What is not bound is known as the free concentration, while the inactive part is known as the bound concentration. Although the amount of boundedness of the drug can change as plasma levels increase, if a linear relationship between the two is present, the free concentration can be determined based on the total concentration. For example, vancomycin is a moderately bound drug where an increase or decrease in its free concentration is based only on the total concentration in the blood [8]. It is known that vancomycin is approximately fifty percent bound to plasma proteins $[5,6]$. Because only the free concentration flows through the body, we need a relationship connecting the two [9]. Together, the free concentration $\left(C_{B f}\right)$ and the bound concentration $\left(C_{B o u n d}\right)$ make up the total concentration of vancomycin in the blood $\left(C_{B l}\right)$. This relationship is given by

$$
C_{B l}=C_{B f}+C_{B o u n d} .
$$

For this particular drug, we assume initially the free concentration is a linear 
function of the total concentration, but we will later assume that a nonlinear relationship exists in order to better fit the model to data (see Section 5) [8]. Because of the boundedness of vancomycin, we are able to write the free concentration as a portion of the total concentration. This is represented in the linear function

$$
C_{B f}=0.51 * C_{B l},
$$

Where the constant, 0.51 , represents the percent of vancomycin not bound to proteins. This relationship is an approximation derived from a clinical study that examined the correlation between the free and bound levels of vancomycin in a group of patients [10].

The rate of infusion, $R_{I}$, represents the quantity of drug entering the body intravenously with respect to time. This is given by Equation (2).

$$
R_{I}= \begin{cases}\frac{D}{T_{I}}, & 0 \leq t \leq T_{I} \\ 0, & t>T_{I}\end{cases}
$$

Here, $D=1 \mathrm{gm}$ is the dosage, and $T_{I}=1 \mathrm{hr}$ is the infusion time.

\subsection{Parameters}

The next part of developing the model involves determining the equations for volumes and flow rates. These provide information regarding how the drug is distributed throughout the different compartments. It is necessary to know the volumes of each compartment in order to examine drug dynamics for the entire system. The equations for the volumes of each compartment as well as blood flow rates are determined from literature. It is necessary that the volumes are based on body weight and height as BMI is based on both factors, therefore allowing us to examine how changes in BMI will affect certain drug factors. These are given in Equation (3). The equations for $V_{B l}$ and $V_{K}$ were from [11], while the equation for $V_{F}$ was from [12] and $V_{G}$ from [13]. For the purposes of modeling we assume the average male has weight $=72 \mathrm{~kg}$ and height $=1.75 \mathrm{~m}$.

$$
\begin{aligned}
V_{B l} & =\frac{13.1(B H * 100)+18.05(B W)-480}{0.5723} \\
V_{K} & =15.4+2.04(B W)+51.8(B H)^{2} \\
V_{F} & =1.36 *\left(\frac{B W}{B H}-42\right) * 1000 \\
V_{G} & =0.0171 *(B W) * 1000 .
\end{aligned}
$$

In order to account for the remaining volume of the other tissues, $V_{O T}$, we will subtract from the total body volume, the volumes above. This is given as 


$$
V_{O T}=B W * 1000-\left(V_{B l}+V_{F}+V_{K}+V_{G}\right) .
$$

From here, the amount of blood flow, and consequently drug flow, through these compartments can be determined [1]. An equation for total flow rate in the body, $Q_{\text {Total }}$, is based upon weight [14]. The flow rates for the individual compartments can be modeled as a fraction of $Q_{\text {Total }}$. These percentages are found in literature and are given in Table 1.

Table 1: Parameter Values Obtained from Literature

\begin{tabular}{cccc}
\hline Parameter & Value & Units & Reference \\
\hline$Q_{\text {Total }}$ & $235 *(B W)^{0.71} * 60$ & $m L / h r$ & {$[14]$} \\
$Q_{F}$ & $0.052 * Q_{\text {Total }}$ & $m L / h r$ & {$[13]$} \\
$Q_{K}$ & $0.19 * Q_{\text {Total }}$ & $m L / h r$ & {$[13]$} \\
$Q_{G}$ & $0.17 * Q_{\text {Total }}$ & $m L / h r$ & {$[13]$} \\
\hline
\end{tabular}

\subsection{Partition Coefficients}

Another important aspect of drug dispersion that must be accounted for is how much of the drug makes it out the tissues. It may be that not all of the drug concentration that enters a tissue via the blood will leave that tissue and be recirculated. Specifically, we must know the tissue's solubility in order to determine what concentration of the drug flowing into the tissue will be trapped there. In order to take this into account, partition coefficients are created. To calculate these partition coefficients, two things from literature must be known, the solubility of the drug in water $\left(S_{w}=0.255\right)$ and the solubility of the drug in n-octanol $\left(K_{o w}=0.000794328\right)$. From here, an algorithm can be used to calculate these partition coefficients, $P_{i}$, for tissue $i$. Blood enters and leaves a compartment in such a way as to maintain equilibrium. Thus, we defined an equilibrium partition coefficient for a tissue, $i$, as $P_{i}$. The fraction of the concentration of the drug leaving each tissue is given in Equation (5).

$$
\frac{C_{i}}{P_{i}} .
$$

Here, $C_{i}$ is the concentration in tissue $i$, and $P_{i}$ is the partition coefficient for that tissue. Notice that the concentration of drug in the blood must be divided by a quantity in such that the concentration of drug flowing into a tissue will be greater than or equal to that which is able to escape. These partition coefficients can be determined using an algorithm developed by Poulin and Krishnan [15, 16]. This is given by 


$$
P_{i}=\frac{\left(\left[S_{o} * N_{t}\right]+\left[\left(S_{w} * 0.7 P_{t}\right)+\left(S_{o} * 0.3 P_{t}\right)\right]+\left[S_{w} * W_{t}\right]\right.}{\left(\left[S_{o} * N_{b}\right]+\left[\left(S_{w} * 0.7 P_{b}\right)+\left(S_{o} * 0.3 P_{b}\right)\right]+\left[S_{w} * W_{b}\right]\right.},
$$

where $i$ corresponds to the specific tissue, and $S_{w}$ and $K_{o w}$ are drug specific parameters [1]. $N_{t}, P_{t}$, and $W_{t}$ represent the fraction of tissue volume that is neutral lipids, phospholipids, and water, respectively. Similarly, $N_{b}, P_{b}$, and $W_{b}$ represent these same fractions in the blood rather than the tissue. The following equation uses $S_{w}$ and $K_{o w}$ to calculate the solubility in n-octanol, $S_{o}$.

$$
S_{o}=K_{o w} * S_{w} .
$$

From this we could determine that $S_{o}=0.000178724$. The partition coefficients calculated from Equation (6) for each tissue in the model is given in Table 2. These values will be considered further in Section 3 .

Table 2: Partition Coefficients

\begin{tabular}{l|l} 
Parameter & Value \\
\hline$P_{F}$ & 0.0009 \\
$P_{K}$ & 0.9767 \\
$P_{G}$ & 0.8898 \\
$P_{O T}$ & 0.9449
\end{tabular}

The last portion of creating the model involves relating all of the information about the flow rates, volumes, and partition coefficients of all the tissues that make up the compartments. Next, the model is condensed to systems of differential equations. This is possible as the drug acts essentially as a rate of flow with respect to time, entering and leaving each compartment.

The blood compartment takes into account the initial infusion quantity along with the recirculation quantities that are emitted from the other compartments. For instance, the portion of drug that escaped the adipose tissue will re-enter the blood and thus circulate through the compartments again. The quantities of excretion are also calculated in order to compare with literature data. The initial conditions are zero as it is assumed there is no initial concentration of vancomycin in the body. The model consists of a system of first-order differential equations being solved simultaneously at different time steps to provide drug concentrations for the antibiotic in the body, given the assumptions in the modeling process. This can then be compared to literature data and the error in the model can be examined [17]. The system of equations that describe this specific system are given by Equation (7). 


$$
\begin{aligned}
V_{F} \frac{d C_{F}}{d t} & =Q_{F}\left(C_{B f}-\frac{C_{F}}{P_{F}}\right) \\
V_{K} \frac{d C_{K}}{d t} & =Q_{K}\left(C_{B f}-\frac{C_{K}}{P_{K}}\right)-k_{u} C_{K} \\
V_{G} \frac{d C_{G}}{d t} & =Q_{G}\left(C_{B f}-\frac{C_{G}}{P_{G}}\right)-k_{f} C_{G} \\
V_{O T} \frac{d C_{O T}}{d t} & =Q_{O T}\left(C_{B f}-\frac{C_{O T}}{P_{O T}}\right) \\
V_{B l} \frac{d C_{B l}}{d t} & =Q_{F} \frac{C_{F}}{P_{F}}+Q_{K} \frac{C_{K}}{P_{K}}+Q_{G} \frac{C_{G}}{P_{G}}+Q_{O T} \frac{C_{O T}}{P_{O T}}-Q_{T o t a l} C_{B f}+R_{I} \\
\frac{d A_{U}}{d t} & =k_{u} C_{K} \\
\frac{d A_{F}}{d t} & =k_{f} C_{G} .
\end{aligned}
$$

Notice in the equations given above, the only parameter values not gathered from data or explicitly calculated are $k_{u}$ and $k_{f}$. These will be estimated in Section 2.3. The schematic for our model is given by Figure 1, and Table 3 summarizes the variables and parameters that have been discussed thus far.

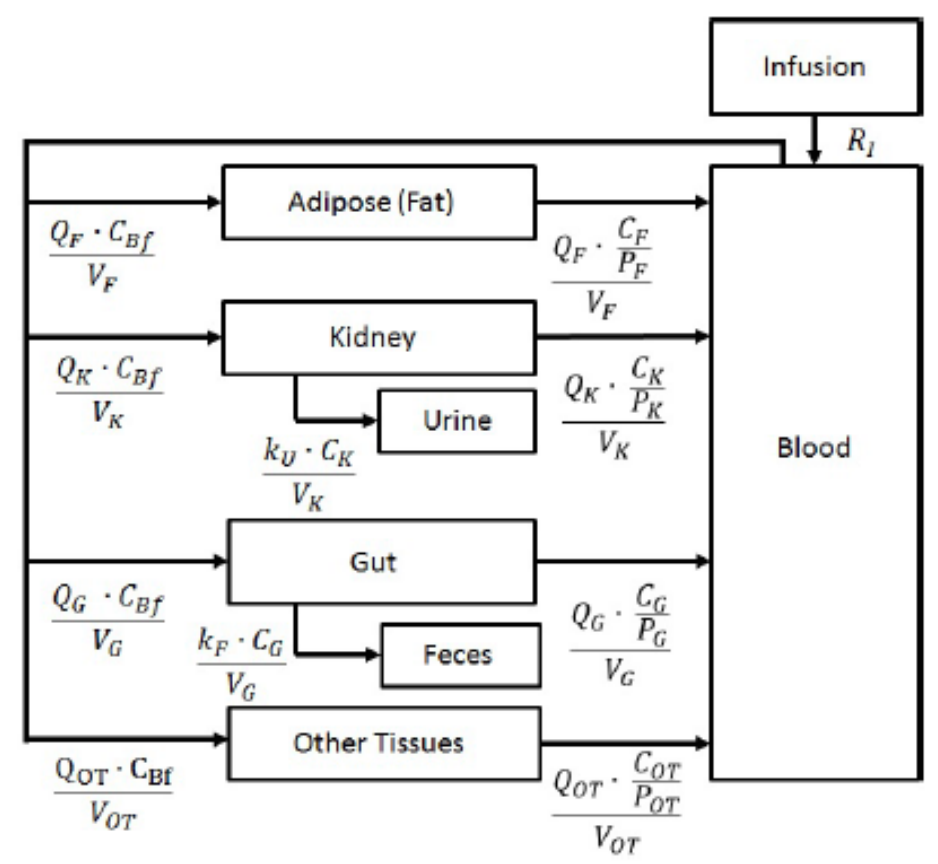

Figure 1 
Table 3: Model Variables and Parameters

\begin{tabular}{l|l|c}
\hline Symbol & Description & Units \\
\hline$C_{i}$ & Concentration of vancomycin in tissue $i$ & $\mathrm{mcg} / \mathrm{mL}$ \\
$C_{B f}$ & Concentration of free vancomycin in the blood & $\mathrm{mcg} / \mathrm{mL}$ \\
$A_{U}$ & Amount of vancomycin in the urine & $\mathrm{mcg}$ \\
$A_{F}$ & Amount of vancomycin in the feces & $\mathrm{mcg}$ \\
$V_{i}$ & Volume of tissue $i$ & $\mathrm{~mL}$ \\
$Q_{i}$ & Flow rate in tissue $i$ & $\mathrm{~mL} / \mathrm{hr}$ \\
$\alpha$ & Infusion Coefficient & dimensionless \\
$t$ & Time & $\mathrm{hr}$ \\
$P_{i}$ & Blood partition coefficient in tissue $i$ & $\mathrm{dimensionless}$ \\
$\mathrm{BW}$ & Body Weight & $\mathrm{mg}$ \\
$\mathrm{BH}$ & Body Height & $\mathrm{mcg} / \mathrm{hr}$ \\
$R_{i}$ & Rate of Infusion & $\mathrm{mcg}$ \\
$D$ & Dose & $\mathrm{hr}$ \\
$T_{i}$ & Length of infusion & $\mathrm{mL} / \mathrm{hr}$ \\
$k_{u}$ & First-order rate constant of urine excretion & $\mathrm{mL} / \mathrm{hr}$ \\
$k_{f}$ & First-order rate constant of feces excretion \\
\hline
\end{tabular}

\subsection{Inverse Problem}

PBPK modeling makes use of known physiological parameters such as blood flow rates through particular tissues, body weight, and organ volumes. It also incorporates estimated parameter values such as rate of excretion in urine $\left(k_{u}\right)$ and feces $\left(k_{f}\right)$. In order to determine which values for these parameters optimize the model so as to best fit its output to the data found in literature, a least squares inverse problem is used. This will compare differences between our literature blood and urine concentrations and those the model outputs for different values of $k_{u}$ and $k_{f}$. This is depicted in Equation (8), where $q=\left[\begin{array}{ll}k_{u} & k_{f}\end{array}\right]$ represents the parameters to be estimated, and $y_{j}$ represents the data at time $j$. The value of the cost function is given as

$$
J(q)=\left(\sum_{j=1}^{N}\left(\frac{\left.\hat{y}_{j}-C_{B l}\left(t_{j}, q\right)\right)}{C_{B l}\left(t_{j}, q\right)}\right)^{2}+\left(\frac{0.8-A_{U}(24)}{A_{U}(24)}\right)^{2}\right) .
$$

The clinical data was obtained by extracting data from a graph using the grabit program in MATLAB $[17,18]$. This data is given in Table 4 . In order to determine which values of $q$ provide the smallest value $J$, a built in MATLAB function, fminsearch, was used. It uses a Nelder-Mead algorithm to estimate parameters based off of an initial guess for the parameters. $J$ is calculated by 
summing the squared differences between the model output given parameters $q$ and the literature data. This allows us to determine the relative error in our model. If the new cost function value is better than the previous, it will continue to guess values in that same direction. It may also choose values in another direction to ensure that it has chosen estimates that minimize the cost function. Once the program reaches a certain threshold, it outputs the optimal parameter values. These optimizing values will then be used in the model.

Table 4: Clinical Data for the Total Concentration of Vancomycin [17]

\begin{tabular}{cc}
\hline $\begin{array}{c}\text { Time }\left(t_{j}\right) \\
(\mathrm{hrs})\end{array}$ & $\begin{array}{c}\text { Clinical Data }\left(C_{B l}\right) \\
(\mathrm{mcg} / \mathrm{mL})\end{array}$ \\
\hline 1 & 63.61 \\
1.08 & 53.67 \\
1.25 & 46.39 \\
1.50 & 38.44 \\
2.00 & 29.17 \\
2.50 & 24.32 \\
3.00 & 21.01 \\
3.40 & 18.14 \\
4.00 & 15.28 \\
5.00 & 12.86 \\
7.00 & 9.35 \\
9.00 & 6.72 \\
13.00 & 4.77 \\
\hline
\end{tabular}

\subsection{Results}

After running the optimization with the initial guess of $q=\left[\begin{array}{ll}10000 & 1000\end{array}\right]$ (values of $k_{u}$ and $k_{f}$ respectively), the fminsearch program determined that the optimal parameter values are $k_{u}=9,993 \frac{\mathrm{mL}}{\mathrm{hr}}$ and $k_{f}=1,691 \frac{\mathrm{mL}}{\mathrm{hr}}$. This gave the cost function a value of $J(q)=1.318$, which represents the sum of squared relative errors across all the data points and excretion levels and implies the model has some error. Figure 2 depicts the urine excretion over the dosing period. This is of importance as this is the primary route of elimination of vancomycin from the body. Clinical data puts urine excretion at $80 \%$ of the initial dose. The model predicted $79.6 \%$ for urine excretion. The percent error between these two is $0.495 \%$. This tells us that the model depiction of drug excretion is very accurate. Any drug remaining in the body is eliminated via the gut, as virtually no amount of the drug is metabolized. 


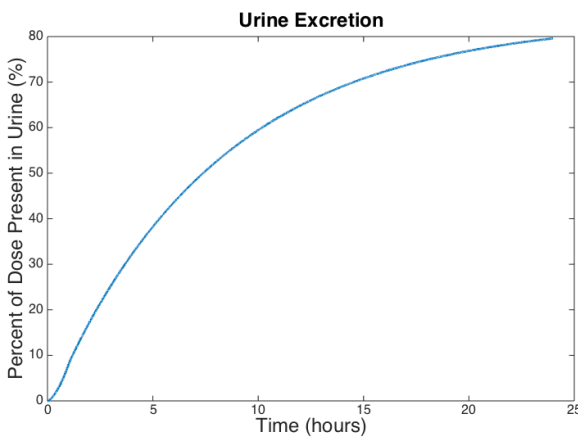

Figure 2: Urine Excretion

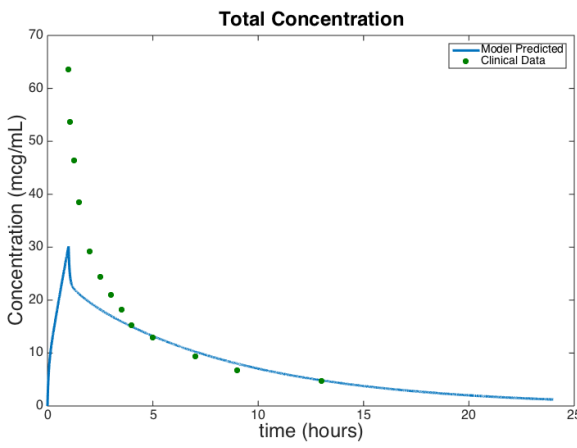

Figure 3: Total Concentration

Figure 3 provides a graphical depiction of the model output for blood concentration versus the clinical data over the twelve hour period following the end of infusion. The percent relative error in point estimates for the total concentration is $47.59 \%$. This is certainly too high a value for relative error. Notice on the graph that the highest peak is not in line with the data. This is the area of least fit for the model. The only area of the data that is in line with model output is at the end of the dosing period.

\section{THE MODEL WITH PARTITION COEFFI- CIENTS AS PARAMETERS}

The fit depicted in Figure 3 in the previous results is not accurate enough to conclude our model is a good representation of how vancomycin acts on the human body. It may be that the partition coefficients in Table 2 are not the true values. This could be due to the fact that the solubility in water and n-octanol were approximated using computer software [6]. In order to test this hypothesis in hopes of improving the model, these partition coefficients will be estimated as parameters, rather than calculated from a formula. In order to determine which of these partition coefficients has the greatest effect on blood concentration, sensitivity analysis was done. In order to do this, the partial of each equation in (7) had to be taken with respect to each of the six parameter values. This allows us to determine the effect each parameter has on the concentration in each compartment. The calculation is done by normalizing the sensitivities using the modified $L_{2}$ norm

$$
\left\|\frac{\partial C_{\text {tissue }}}{\partial q_{j}}\right\|_{2}=\left[\frac{1}{t_{f}-t_{0}} \int_{t_{0}}^{t_{f}}\left(\frac{\partial C_{\text {tissue }}}{\partial q_{j}}\right)^{2} d t\right]^{\frac{1}{2}} \frac{q_{j}}{\max C_{\text {tissue }}} .
$$

A trapezoidal approximation was used to evaluate the integral in (9) [1]. Using Equation (9), results of these calculations are depicted below. 


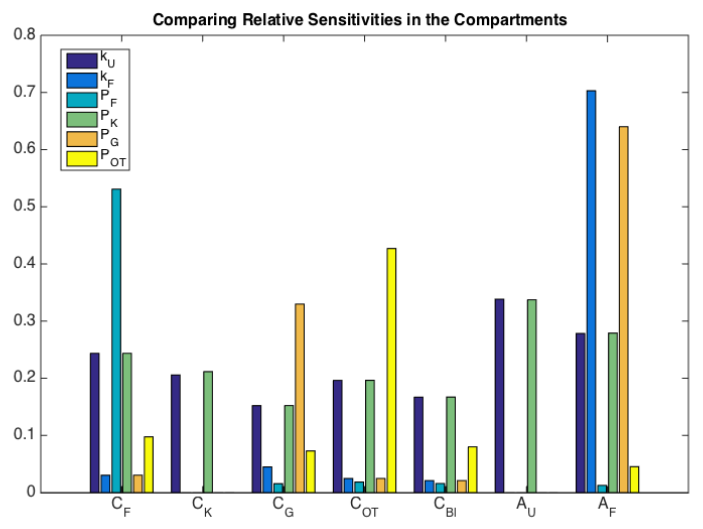

Figure 4: Sensitivity Analysis

The results showed no particular partition coefficient dominated the concentration of the drug in all of the compartments. For instance, in Figure (4) it is clear that partition coefficient $P_{F}$ had the most effect on the fat compartment $C_{F}$. However, in the gut compartment, $C_{G}$, the partition coefficient $P_{G}$ had the most effect. This same trend can be seen with all of the compartments and partition coefficients. Thus, all will need to be estimated as parameters. We use the same cost function given by Equation (8). Our input parameters are given by $q=\left[\begin{array}{llllll}P_{F} & P_{K} & P_{G} & P_{O T} & k_{u} & k_{f}\end{array}\right]$. The result of estimating the partition coefficients as parameters along with the rates of excretion in the urine and the feces is depicted in Figure 5. The minimization resulted in the parameter estimates given in Table 5 .

Table 5: Partition Coefficients

\begin{tabular}{l|l} 
Parameter & Value \\
\hline$P_{F}$ & 0.00034 \\
$P_{K}$ & 1.345 \\
$P_{G}$ & 0.143 \\
$P_{O T}$ & 0.757 \\
$k_{u}$ & 5,981 \\
$k_{f}$ & 12,066
\end{tabular}




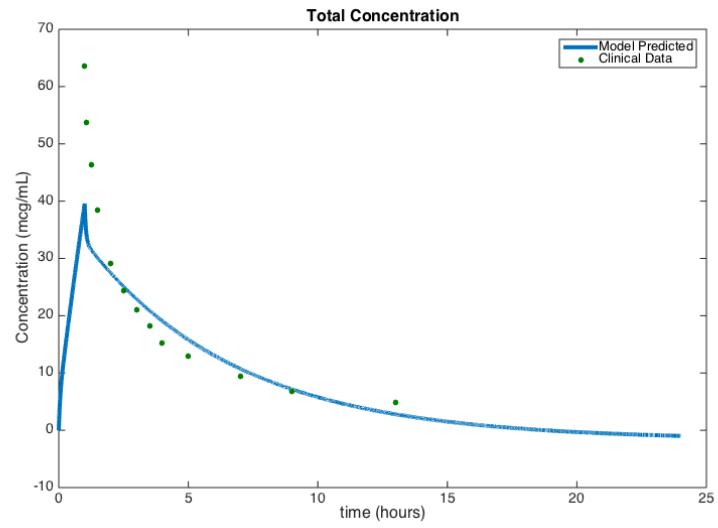

Figure 5: Total Concentration with Partition Coefficients as Parameters

Although a better fit than previously, it is clear that this does not solve the problem of the peak being too low. The next section will discuss alternative changes to be made to the model in order to better improve the fit.

\section{THE MODEL WITH AN INFUSION COEF- FICIENT}

As noted in the previous section, our model falls short in predicting the accurate peak of vancomycin concentration in the blood. For this reason, a new parameter $\alpha$ is introduced into the model as was done in [1]. This parameter acts as an infusion coefficient, slowing the rate of drug dispersion throughout the compartments during the infusion period. The infusion coefficient, $\alpha$, is given as

$$
\alpha=\left\{\begin{array}{cl}
\alpha_{I}, & 0<t \leq T_{I} \\
1, & T_{I}<t<12
\end{array} .\right.
$$

This makes sense biologically as the quantity of vancomycin traveling through the various compartments may not reach its maximum until the entire dose of the drug has been administered. In addition, the way in which the drug binds during infusion may be different than the way it binds once the entire dose is administered. This changed the system of Equations in (7) by replacing $C_{B f}$ by $\alpha * C_{b f}$ where $\alpha=1$ after the infusion period and is an unknown parameter during the infusion period, which we will estimate. Now $q=\left[\begin{array}{lllllll}P_{F} & P_{K} & P_{G} & P_{O T} & k_{u} & k_{f} & \alpha\end{array}\right]$, with an initial guess of $\alpha=0.35$. The minimization algorithm was run again to determine the optimal parameter estimates. The estimate for $\alpha$ was given as 0.46 , and $k_{u}=4,998 \frac{m L}{h r}$ and $k_{f}=5,968 \frac{m L}{h r}$. The solution curve for the model is plotted with the data in Figure 6 . The ending cost function value was $J=0.44$. The percent relative error in point estimates for total concentration was approximately $20 \%$. 


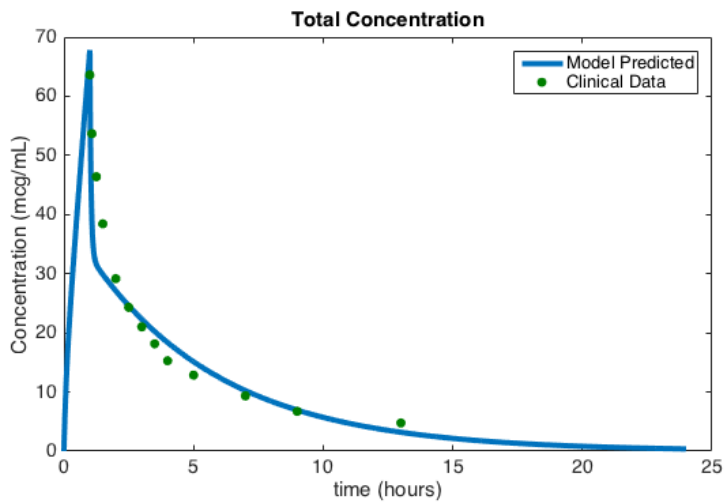

Figure 6: Total Concentration with Infusion Coefficient

Notice that although the addition of $\alpha$ as a parameter into the model did allow for the correct concentration peak, during the times following the peak but before the blood concentration levels out, the model does not fit the data well. A nonlinear excretion in the urine was implemented to try to fix this problem but the effects were negligible. The following section discusses the next steps taken to better the model.

\section{THE MODEL WITH NONLINEAR BLOOD CONCENTRATIONS}

From the results given in Figure 6 it is clear that the model output for the peak blood concentration as well as the ending blood concentrations match the literature data fairly well. The problem is believed to be in the linearity of the model during the middle of the timespan. One possible explanation for this is that the binding of the drug is not entirely linear. That is, Equation (1) is a linear approximation to a nonlinear relationship between the free and bound concentrations of the drug in the blood. To test this hypothesis, a nonlinear Michaelis-Menten equation is used to model the bound concentration. This equation is given by

$$
C_{\text {Bound }}=\frac{B_{m} C_{B f}}{K_{d}+C_{B f}} .
$$

Here, $B_{m}$ represents the blood receptor content and $K_{d}$ the dissociation constant [19]. Because the total blood concentration is the sum of the free and bound concentrations, using Equation (10) we have

$$
C_{B l}=C_{B f}+\frac{B_{m} C_{B f}}{K_{d}+C_{B f}} .
$$

As seen in Equation (11), it is not necessary to directly calculate the bound concentration of the drug in order to study the total and free concentrations 
in the blood. Algebraic manipulation results in the following equation, which provides the free concentration as a function of $C_{B l}, B_{m}$, and $K_{d}$

$$
C_{B f}=\frac{C_{B l}-B_{m}-K_{d}+\sqrt{\left(B_{m}+K_{d}-C_{B l}\right)^{2}+4 K_{d} C_{B l}}}{2} .
$$

Notice there are now two additional parameters $B_{m}$ and $K_{d}$. Using Equation (12) for the free concentration in the modified version of the model with the parameter $\alpha$, we estimate the parameters. We use $q=\left[\begin{array}{llllll}P_{F} & P_{K} & P_{G} & P_{O T} & B_{m}\end{array}\right.$ $\left.\begin{array}{llll}K_{d} & k_{u} & k_{f} & \alpha\end{array}\right]$ in the cost function (Eq. (8)). The results of doing this are given by Figure 7. It is clear that the nonlinear blood concentration approach provides the most accurate model. To verify, the cost function value after the minimization is $J=0.034$. This is the smallest value of $J$ so far in the model development.

Table 6: Results

\begin{tabular}{c|c|c}
\hline $\begin{array}{c}\text { Literature Concentrations }[17] \\
(\mathrm{mcg} / \mathrm{mL})\end{array}$ & $\begin{array}{c}\text { Model Approximations } \\
(\mathrm{mcg} / \mathrm{mL})\end{array}$ & $\begin{array}{c}\text { Percent Error } \\
\%\end{array}$ \\
\hline 63.61 & 62.12 & 2.3 \\
53.67 & 53.60 & 0.2 \\
46.39 & 44.84 & 3.3 \\
38.44 & 37.84 & 1.6 \\
29.17 & 28.92 & 0.8 \\
24.32 & 23.38 & 3.9 \\
21.012 & 19.78 & 5.9 \\
18.15 & 17.33 & 4.5 \\
15.28 & 15.56 & 1.8 \\
12.86 & 13.07 & 1.6 \\
9.35 & 9.83 & 5.1 \\
6.73 & 7.50 & 11.5 \\
4.77 & 4.34 & 9.0 \\
\hline \hline Exact Urine Excretion & Approximate Urine Excretion & Percent Error \\
$(m c g / m L)$ & $(m c g / m L)$ & $\%$ \\
0.8 & 0.791 & $1.125 \%$ \\
\hline
\end{tabular}




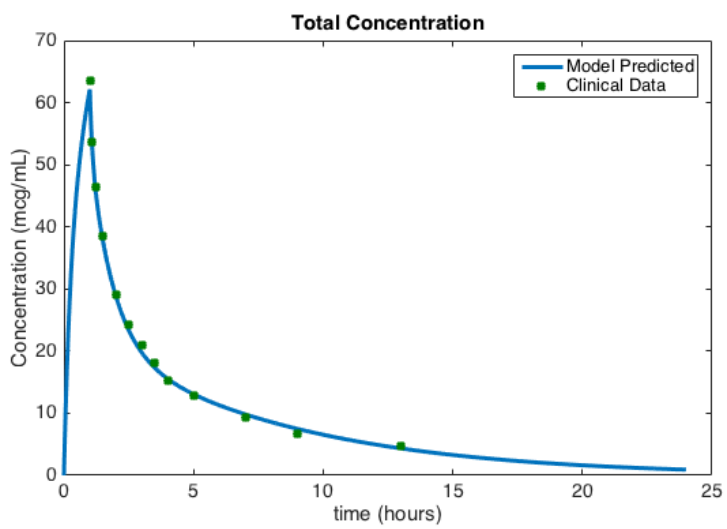

Figure 7: Nonlinear Total Concentration

There are other important pieces of data resulting from the final model that will be useful in future analysis. The model provided us with the area under the curve (AUC), which is 224. This information is important as it relates again to renal toxicity. Having too large an area could increase the risk of renal failure or other harmful side effects of the drug. The ratio between AUC and minimum inhibitory concentration (MIC) is also significant in trying to determine the most effective dosing. Specifically, it has been related to vancomycin's effectiveness in treating staph infections [20]. The lowest concentration level given by the model is in line with the lowest blood concentration as seen in Table 6. This value is of importance as the next dose would begin at the end of the first dosing period in such a way as to ensure that this level does not fall below the MIC in order to prevent the development of resistant bacteria. This is also the area of focus when adjusting body mass. Specifically, one may be interested in whether or not differing BMIs will cause blood concentration levels to drop below the MIC.

\section{CONCLUSIONS}

The development of a model that provides comparable information to measure the absorption, distribution, and elimination of vancomycin provides a solid base for continued research regarding this drug. Specifically, it allowed for the examination into the physiological effects body mass index has on these drug factors in work by Dr. Michele Joyner [2]. Because compartment volume is based off body mass, we are able to adjust these volumes according to changes in body mass and produce model predictions based on differing body masses. We may also be interested in differences between male and female absorption. These could also be related to compartment volume as the average body mass for males and females differ from one another. Another aspect of future work will be in improving the accuracy of the model even further. One way is in evaluating 
the reliability of the parameters estimated. Some of the parameters gathered from literature did not provide useful information when input into the model. For instance, the solubility in water and n-octanol:water of vancomycin was gathered from literature but did not provide for biologically plausible partition coefficients that allowed for a good fit of the model to the data. For vancomycin, the lack of data regarding its chemical properties as well as data on the blood concentrations after infusion posed some problems in developing an accurate model. Throughout the development of the model until its final version, the parameters being estimated changed as the model was modified. In the future, statistical analysis could be implemented to determine confidence intervals for the parameters being estimated. This would provide information about the degree to which the model could vary based on changing initial parameters values. Overall, modifying a PBPK model for ertapenem resulted in a reliable model of the absorption, distribution, and elimination of vacomycin [1]. The model produced results for blood concentrations with less than a total of $2.63 \%$ error in point estimates and less than $1.1 \%$ error in urine excretion levels. 


\section{References}

[1] Whitney Forbes, Michele Joyner, Michelle Maiden, Cammey Manning, and Ariel Nikas. A physiologically-based pharmacokinetic model for the anitibiotic ertapenem. Mathematical Biosciences and Engineering, 2016.

[2] Michele Joyner. Modeling the effect of body mass index on blood concentration levels for three different antibiotics. Presented at 2016 SIAM SEAS, March 2016.

[3] Catherine Liu, Arnold Bayer, Sara E. Cosgrove, Robert S. Daum, Scott K. Fridkin, Rachel J. Gorwitz, Sheldon L. Kaplan, Adolf W. Karchmer, Donald P. Levine, Barbara E. Murray, Michael J. Rybak, David A. Talan, and Henry F. Chambers. Clinical practice guidelines by the infectious diseases society of america for the treatment of methicillin-resistant staphylococcus aureus infections in adults and children. Clinical Infectious Diseases, 2011.

[4] Michael Shnayerson and Mark Plotkin. The Killers Within:The Deadly Rise of Drug-Resistant Bacteria. Back Bay Books, 0316713317, 2003.

[5] Michael J. Rybak. The pharmacokinetic and pharmacodynamic properties of vancomycin. Clinical Infectious Diseases, 42(Supplement 1):S35-S39, 2006.

[6] Drug Bank. Vancomycin. http://www.drugbank.ca/drugs/DB00512, 2014.

[7] Kerry Estes and Hartmut Derendorf. Comparison of the pharmacokinetic properties of vancomycin, linezolid, tigecyclin, and daptomycin. European Journal of Medical Research, pages 533-543, 2010.

[8] Michael Rybak, Ben Lomaestro, John C. Rotschafer, Robert Moellering, William Craig, Marianne Billeter, Joseph R. Dalovisio, and Donald P. Levine. Therapeutic monitoring of vancomycin in adult patients: A consensus review of the american society of health-system pharmacists, the infectious diseases society of america, and the society of infectious diseases pharmacists. American Journal of Health-System Pharmacy, 66(1):82-98, 2009 .

[9] Sun He. Serum protein-binding characteristics of vancomycin. Antimicrobial Agents and Chemotherapy, 37(5):1132-1136, 1993.

[10] Jill M. Butterfield, Nimish Patel, Manjunath P. Pai, Thomas G. Rosano, George L. Drusano, and Thomas P. Lodise. Refining vancomycin protein binding estimates: Identification of clinical factors that influence protein binding. Antimicrobial Agents and Chemotherapy, 55(9):4277-4282, 2011.

[11] P.S. Price, R.B. Conolly, C.F. Chaisson, E.A. Gross, J.S. Young, E.T. Mathis, and D.R. Tedder. Modeling interindividual variation in physiological factors used in PBPK models of humans. Crit. Rev. Toxicol., 33(5):469503, 2003. 
[12] Henry Kvist, Badrul Chowdhury, U. Grangård, Ulf Tylen, and L. Sjöström. Total and visceral adipose-tissue volumes derived from measurements with computed tomography in adult men and women: predictive equations. Am. J. Clin. Nutr., 48(6):1351-1361, 1988.

[13] S. Pilari and W. Huisinga. Lumping of physiologically-based pharmacokinetic models and a mechanistic derivation of classical compartmental models. J. Pharmacokinet. Phar., 37, 2010.

[14] Giovanni de Simone, Richard B. Devereux, Stephen R. Daniels, GianFrancesco Mureddu, Mary J. Roman, Thomas R. Kimball, Rosanna Greco, Sandra Witt, and Franco Contaldo. Stroke volume and cardiac output in normotensive children and adults: assessment of relations with body size and impact of overweight. Circulation, 95(7):1837-1843, 1997.

[15] Patrick Poulin and Kannan Krishnan. An algorithm for predicting tissue:blood partition coefficients of organic chemicals from n-octanol:water partition coefficient data. J. Toxicol. Env. Health, 46:117-129, 1995.

[16] Patrick Poulin and Kannan Krishnan. A biologically-based algorithm for predicting human tissue:blood partition coefficients of organic chemicals. Human and Experimental Toxicology, 14:273-280, 1995.

[17] Daniel Healy, Ron Polk, Michelle Garson, David Rock, and Thomas Comstock. Comparison of steady-state pharmacokinetics of two dosage regimens of vancomycin in normal volunteers. Antimicrobial Agents and Chemotherapy, 31:393-397, 1987.

[18] MATLAB. version 7.13.0.564 (R2011b). The MathWorks Inc., Natick, Massachusetts, 2011.

[19] D. Plowchalk and J. Teeguarden. Development of a physiologically based pharmacokinetic model for estradiol in rats and humans: A biologically motivated quantitative framework for evaluating responses to estradiol and other endocrine-active compounds. Toxicol. Sci., 69:60-78, 2002.

[20] Natasha E. Holmes, John D. Turnidge, Wendy J. Munckhof, J. Owen Robinson, Tony M. Korman, Matthew V. N. O'Sullivan, Tara L. Anderson, Sally A. Roberts, Sanchia J. C. Warren, Wei Gao, Benjamin P. Howden, and Paul D. R. Johnson. Vancomycin auc/mic ratio and 30-day mortality in patients with staphylococcus aureus bacteremia. Antimicrobial Agents and Chemotherapy, 57(4):1654-1663, 2013. 